\title{
CIRED 2015 - Internationale Konferenz über elektrische Verteilnetze
}

\author{
W. Tenschert OVE
}

Online publiziert am 5. November 2015

(c) Springer Verlag Wien 2015

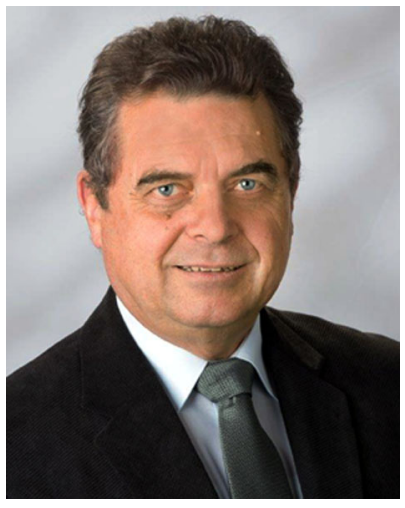

Dipl.-Ing. Dr. W. Tenschert
Die internationalen Konferenzen der CIRED haben sich zu einer der weltweit wichtigsten Plattformen über den Stand der Technik und die laufende Forschungs- und Entwicklungsarbeit bei elektrischen Verteilnetzen entwickelt. Nach 2013 in Stockholm fand die 23. CIREDKonferenz vom 15. bis 18 . Juni 2015 im Convention Center von Lyon in Frankreich statt.

1.287 Teilnehmer aus 61 Ländern nutzten die vier Tage in Lyon zum Wissens- und Erfahrungsaustausch. Von mehr als 1.600 zur Konferenz eingereichten Beiträgen wurde ca. die Hälfte in einem umfassenden Evaluierungsprozess, in den auch zahlreiche österreichische Experten eingebunden waren, akzeptiert. Letztendlich wurden 754 Beiträge, davon 20 aus Österreich, auf der Konferenz präsentiert.

Wie schon in den vergangenen Jahren war die Konferenz in sechs fachliche Teilbereiche (Sessions) gegliedert:

(1) Netzkomponenten

(2) Power Quality und elektromagnetische Verträglichkeit

(3) Betrieb, Steuerung und Schutz

(4) Dezentrale Energieressourcen und Energieeffizienz

(5) Netzentwicklung

(6) Strommärkte und Regulierung

Am ersten Tag fanden sechs Tutorials zu ausgewählten Themen in Verteilnetzen sowie die Eröffnungsvorträge statt. An den drei Haupttagen der Konferenz wurden die Beiträge der einzelnen Sessions präsentiert. Dafür standen teilweise zeitgleich abgehaltene PlenumVorträge, Round-table-Diskussionen, Forschungs- und Innovationsforen sowie Postersessions statt. Insbesondere die Postersessions, bei denen die Autoren ihre Beiträge im direkten Gespräch mit interessierten Experten erläutern konnten, fanden großen Anklang. Eine umfassende Fachausstellung mit mehr als 110 namhaften Herstellern ergänzte das Programm. Im Anschluss an die Konferenz konnten wieder bedeutende technische Anlagen bzw. Herstellerwerke in der Umgebung von Lyon besichtigt werden.

Auch 2015 in Lyon war die Beteiligung Österreichs sowohl nach Beiträgen, Konferenzteilnehmern als auch Konferenzmitgestaltern überproportional groß. Besonderer Dank gilt somit den österreichischen Berichterstattern, den Vertretern der österreichischen Univer- sitäten sowie allen Mitgliedern und Freunden des Österreichischen Nationalkomitees der CIRED, die zum Erfolg der CIRED-Konferenz 2015 in Lyon beigetragen haben.

Im Directing Commitee Meeting im Anschluss an die Konferenz wurde Dipl.-Ing. Theodor Connor (Siemens) für weitere zwei Jahre als Vorsitzender bestätigt. Ebenso bestätigt wurde Prof. Emmanuel de Jaeger als Vorsitzender des Technischen Komitees.

Mit Ende der CIRED-Konferenz 2015 verabschiedete sich auch Dipl.-Ing. Herbert Haidvogl offiziell von der CIRED. Als langjähriger Vorsitzender des Directing Committee hat er maßgeblich die Entwicklung der CIRED geprägt. Ein herzliches Dankeschön an Herbert Haidvogl, auch für sein Wirken als Vorsitzender des Österreichischen Nationalkomitees der CIRED.

Für diejenigen, die an der Konferenz nicht teilnehmen konnten, bietet das Österreichische Nationalkomitee wieder zwei InfoNachmittage mit Präsentationen über die aktuellen Trends der sechs Sessions an:

\section{- In Wien:}

Zeit: Dienstag, 26. Jänner 2016, 14:00 Uhr

Ort: OVE Österreichischer Verband für Elektrotechnik, Eschenbachgasse 9, 1010 Wien

\section{- In Innsbruck}

Zeit: Dienstag, 2. Februar 2016, 14:00 Uhr

Ort: Hotel Grauer Bär, Universitätsstraße 5-7, 6020 Innsbruck

Weiters möchte ich Sie auf die nächsten CIRED-Veranstaltungen aufmerksam machen:

\section{- CIRED-Workshop 2016 - Helsinki, Finnland}

14.-15. Juni 2016, Marina Congress Centre

Electrical Networks for Society and People. Business models and their technical solutions enabling smart distribution grids

\section{- CIRED-Konferenz 2017 - Glasgow, Schottland, UK}

12.-15. Juni 2017, Scottish Exhibition \& Conference Centre (SECC)

Die nachfolgenden Beiträge über die sechs Sessions stellen eine Kurzfassung der Inhalte und Tendenzen dar und sollen zu einer intensiveren Befassung mit den Beiträgen der CIRED-Konferenz anregen.

Weiterführende Informationen oder Ansprechpartner finden Sie auf den Websites:

\section{www.cired.at}

www.cired2015.org

www.cired2016-workshop.org

www.cired-2017.org

Tenschert, Walter, Netz Oberösterreich GmbH, Neubauzeile 99, 4030 Linz, Österreic (E-Mail: walter.tenschert@netzgmbh.at) 